\title{
Comparison of the Effectiveness of Narrative Therapy and Emotionally Focused Therapy on Family Cohesion of Veterans' Wives; A Case Study of Khorramabad City
}

\section{ART ICLE INF O}

\section{Article Type}

Original Research

\section{Authors}

Abbasi S. ${ }^{1} \mathrm{PhD}$,

Dokaneheifard F* $P h D$,

Shafiabady A. ${ }^{2} P h D$

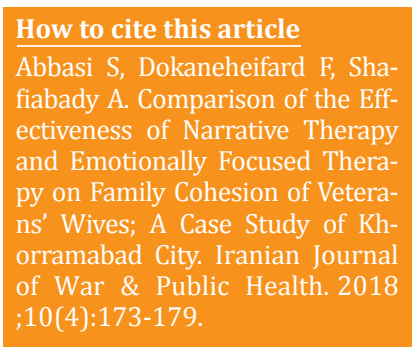

*Consultation Department, Educational Sciences \& Consultation Faculty, Roodehen Branch, Islamic Azad University, Roodehen, Iran

${ }^{1}$ Consultation Department, Educational Sciences \& Consultation Faculty, Roodehen Branch, Islamic Azad University, Roodehen, Iran

${ }^{2}$ Consultation Department, Psychology \& Educational Sciences Faculty, Allame Tabatabaii University, Tehran, Iran

\section{*Correspondence}

Address: Consultation Department, Islamic Azad University, Academic Complex, Roodehen, Iran. Postal Code: 3973188981

Phone: +98 (21) 76509324

Fax: +98 (21) 76509324

f.dokaneifard@riau.ac.ir

\section{Article History}

Received: February 14, 2018

Accepted: May 16, 2018

ePublished: October 11, 2018

\section{A B S T R A C T}

Aims Despite passing of years since the end of the war, veterans are still experiencing a lot of physical and psychological problems, which can seriously affect well-being of their wives and, consequently, family function. The aim of this study was to compare the effectiveness of narrative therapy and emotionally focused therapy on family cohesion of veterans' wives in Khorramabad. Materials \& Methods The present semi-experimental research was carried out in 2017 with two experimental and one control groups and a three-month follow-up in the community of veterans' wives in Khorramabad, in which 60 subjects were selected by purposive sampling method and randomly divided into two experimental and one control groups (each group included 20 subjects). The first experimental group received 10 narrative therapy sessions (120 minutes for each session) and the second group received 10 emotionally focused therapy (120 minutes for each session) and the control group was placed in waiting list. The Samani family cohesion questionnaire was used as a research tool. The data were analyzed by SPSS 20 software, using repeated measures ANOVA and Bonferroni post hoc test.

Findings In the post-test and follow-up phase, there was a significant difference in family cohesion scores between narrative therapy and emotionally focused therapy groups in comparison with the control group $(\mathrm{p}<0.01)$, and both treatments had the same efficacy $(\mathrm{p}>0.05)$.

Conclusion Narrative therapy and emotionally focused therapy are effective in improving family cohesion in veterans' wives.

Keywords Narrative Therapy; Emotionally Focused Therapy; Family Cohesion; Veteran

\section{CIT A T I O N L IN KS}

[1] The effectiveness of hope therapy on quality of life in wives of veterans with post traumatic stress ... [2] Psychological sequellae of combat violence: A review of the ... [3] Stress disorders among vietnam veterans ... [4] Effectiveness of training life skills on the quality of life of spouses of veterans with affective ... [5] Systemic family therapy: From theory ... [6] Cross-cultural applicability of FACES ... [7] FACES IV and the circumplex model: Validation ... [8] Acceptance and commitment therapy for treatment-resistant posttraumatic stress disorder: A case ... [9] The effectiveness of narrative therapy on increasing couples intimacy and its dimensions ... [10] The meaning of others: Narrative studies of ... [11] The narrative construction of intimacy and affect in relationship stories: Implications for ... [12] “Good Enough Stories": Helping couples invest in one another's ... [13] Dating is hard work: A narrative approach to understanding sexual and romantic ... [14] The effectiveness of group narrative therapy on the habitual life and happiness of the elderly in ... [15] The effect of couple therapy narrative on family function of ... [16] How avoidant attachment influences subjective well-being ... [17] Dissociable effects of conscious emotion regulation strategies on explicit ... [18] A secure base: Parent-child attachment and healthy human ... [19] Integrating sex and attachment in emotionally ... [20] Attachment styles, relationship satisfaction ... [21] Perceptions of attachment style and marital quality ... [22] Emotion-focused ... [23] The effectiveness of emotional focused couple therapy on satisfaction and ... [24] Predicting change in marital satisfaction throughout ... [25] An investigation of effectiveness of the emotionally focused couple ... [26] Marital satisfaction and break-ups differ across on-line and offline meeting ... [27] Developing a family cohesion scale for Iranian ... [28] Reflections on narrative practice: Essays ... [29] The practice of emotionally focused marital therapy ... [30] The effects of integrative reminiscence on depressive ... [31] The effectiveness of couples therapy based on acceptance ... 


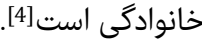

انسجام و انطباق، دو جنبه رفتار زناشويى و خانو خانوادگى است كه

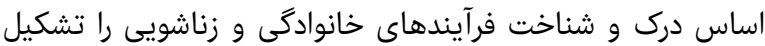

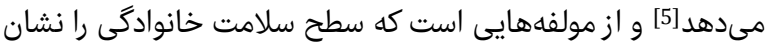

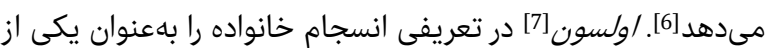

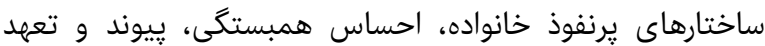

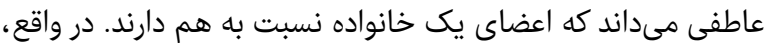

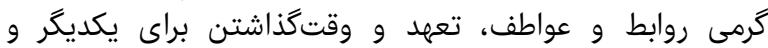

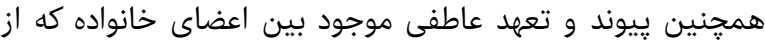

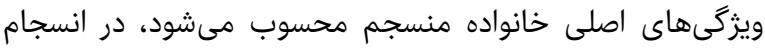

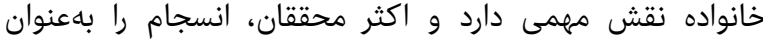

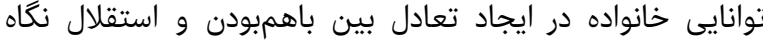

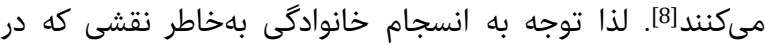

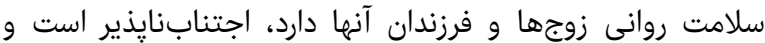

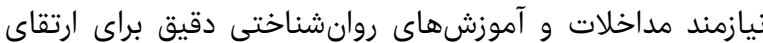

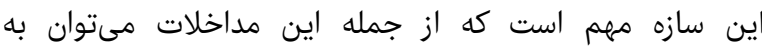

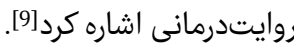

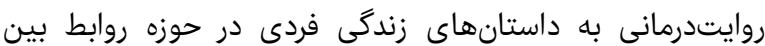

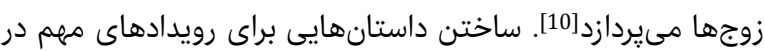

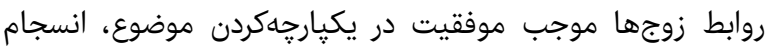

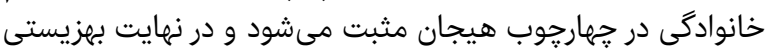

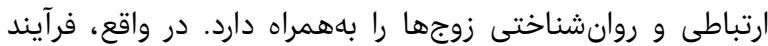

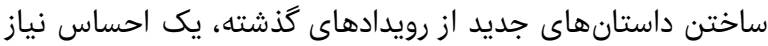

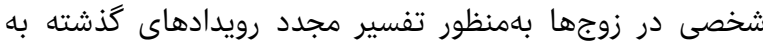

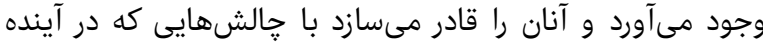

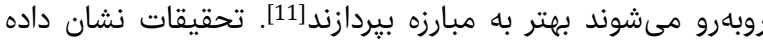

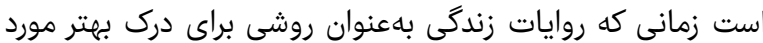

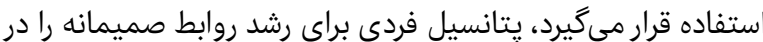

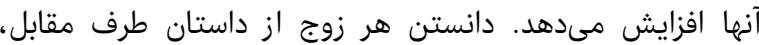

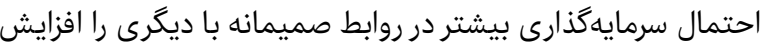

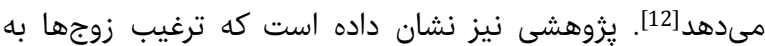

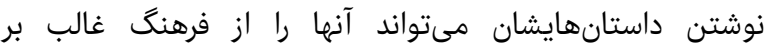

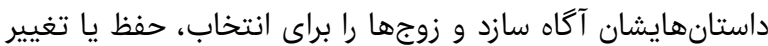

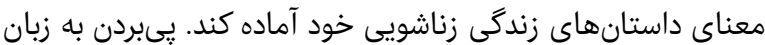

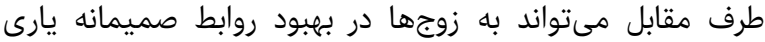

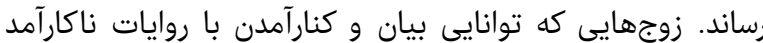

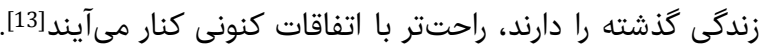

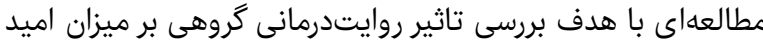

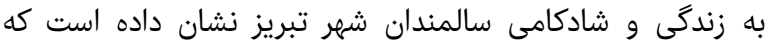

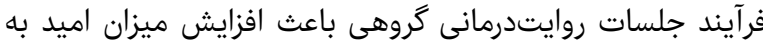

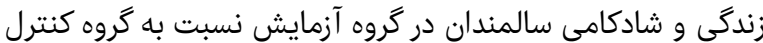

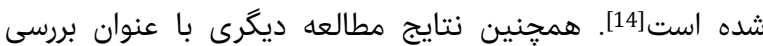

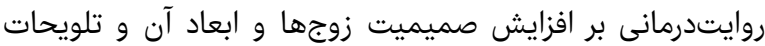

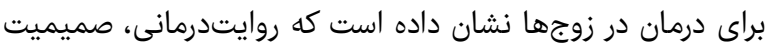

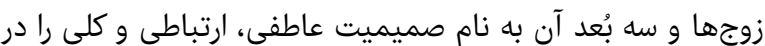

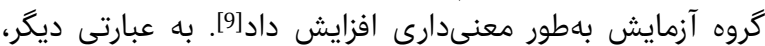

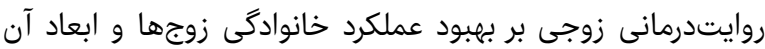

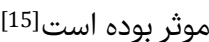

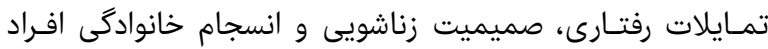

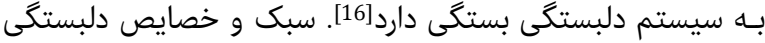

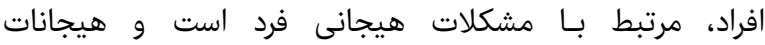
تنظيمنشـده بـا اشكال برجستهـ آسـيب روانى همراه است.

\section{مقايسه اثربخشى روايتدرمانى و درمان هيجانمحور بر انسجام خانوادگى همسران جانباز؛ مطالعه موردى شهرستان خرم آباد}

سميه عباسى PhD كروه مشاوره، دانشكده علوم تربيه عربيتى و مشاوره، واحد رودهن، دانشگاه آزاد

اسلامى، رودهن، ايران

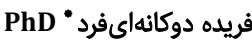

كروه مشاوره، دانشكده علوم تربيته

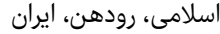

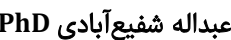
كروه مشاوره، دانشكده روانشآناسى و علوم تربيتى، دانشكاه علامه طباطبايى،

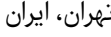

جكيده

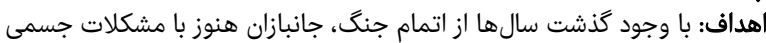

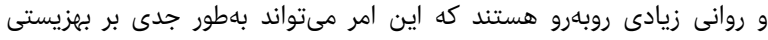

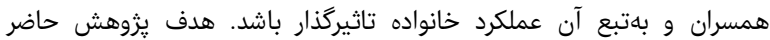

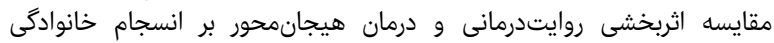

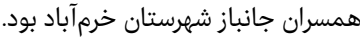

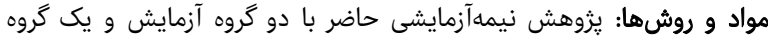

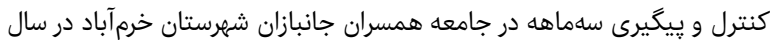

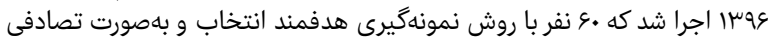

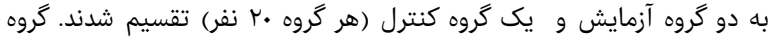

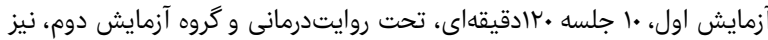

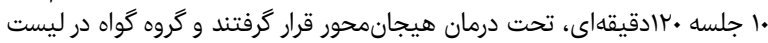

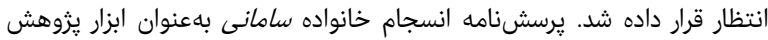

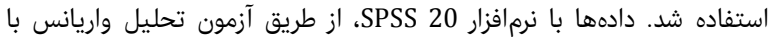

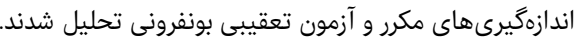

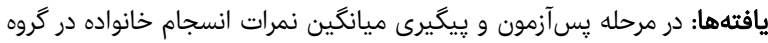

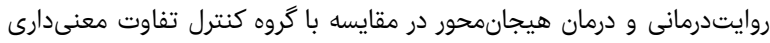

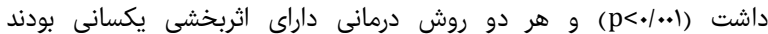

(p>・/・a)

نتيجهيرى: روايتدرمانى و درمان هيجانمحور بر بهبود انسجام خانواده

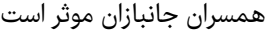
كليدوازهها: روايتدرماني، درمان هيجانمحور، انسجام خانواده، جانباز

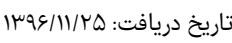

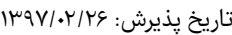

"نويسنده مسئول:

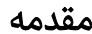

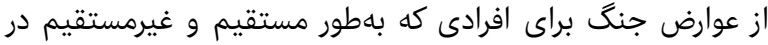

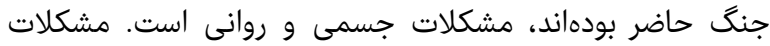

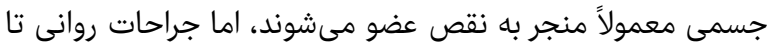

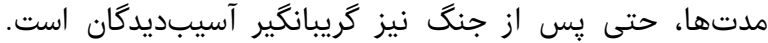

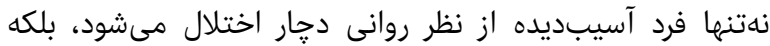

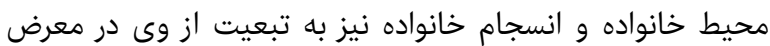

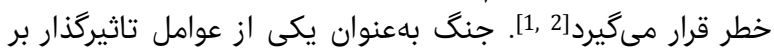

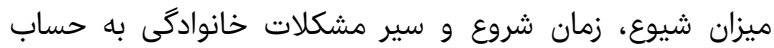

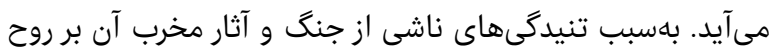

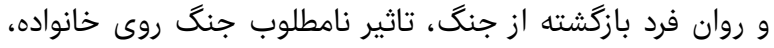

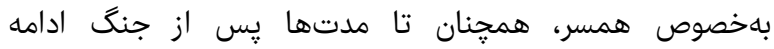

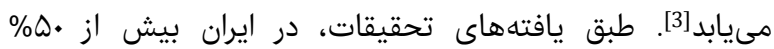

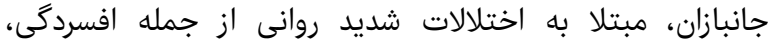

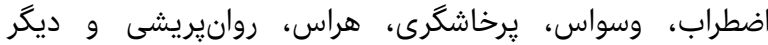
اختلالات روانى هستند كه نتيجه اين اختلالات، كاهش انسران انسجام 


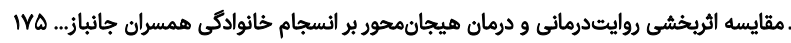

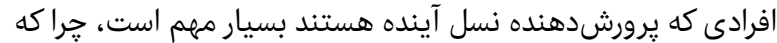

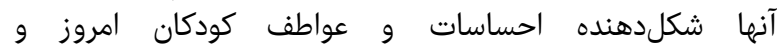

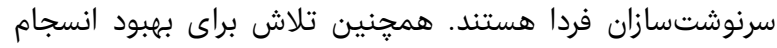

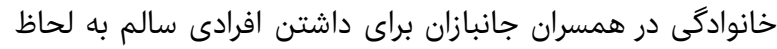

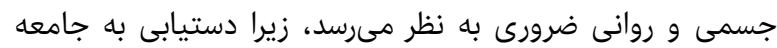

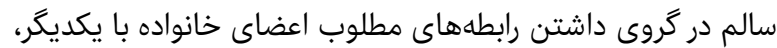

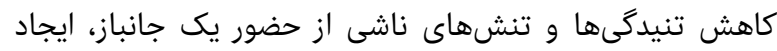

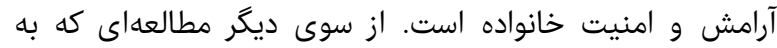

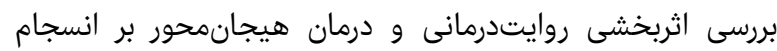

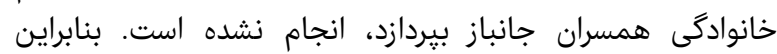

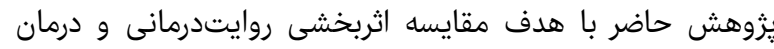

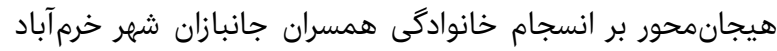

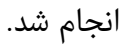

\section{مواد و روشها}

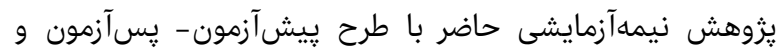

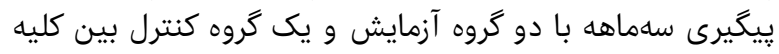

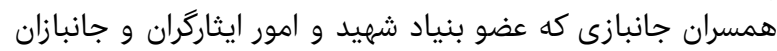

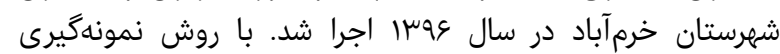

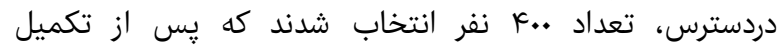

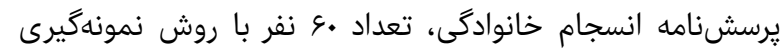

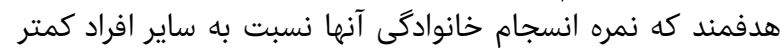

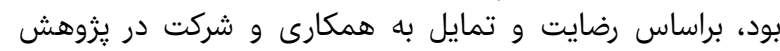

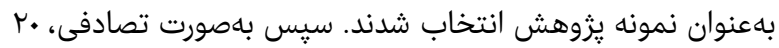

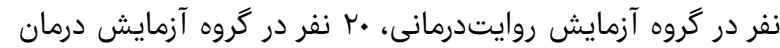

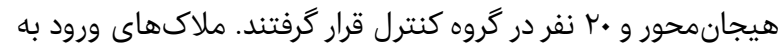

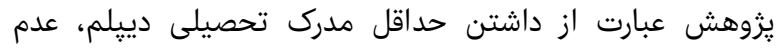

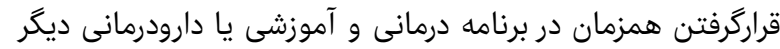

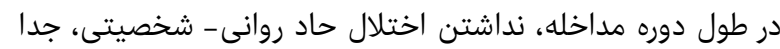

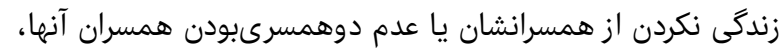

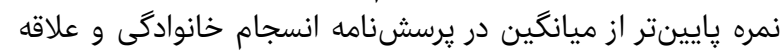

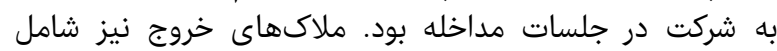

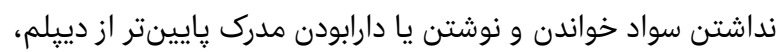

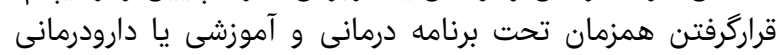

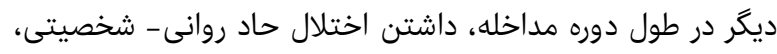

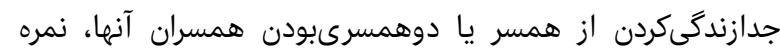

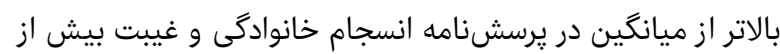
دو جلسه در جلسات مداخله بود.

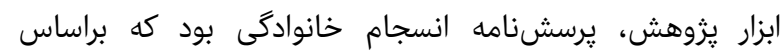

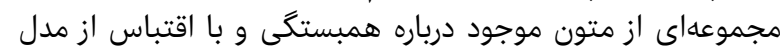

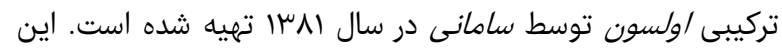

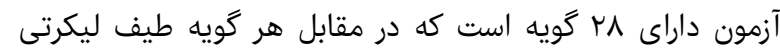

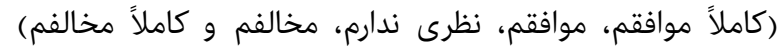

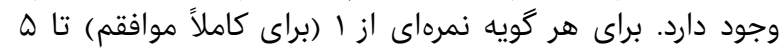

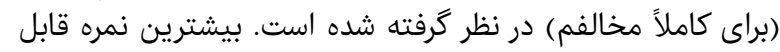

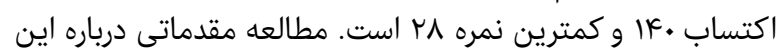

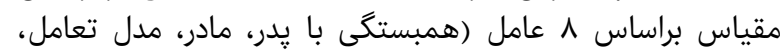

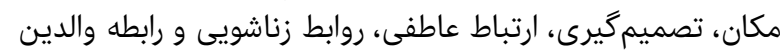

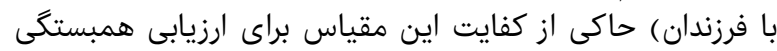

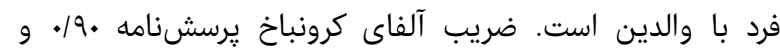

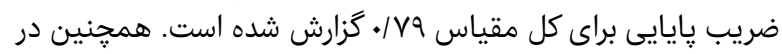

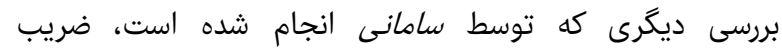

ناتوانى در نظمجويى هيجانى، منشا رفتارهـاى مشـكلآفرين

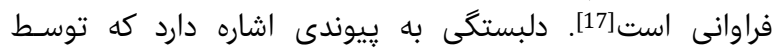

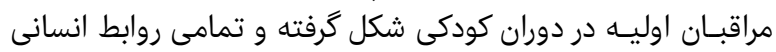

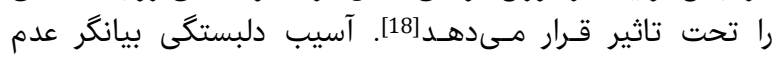

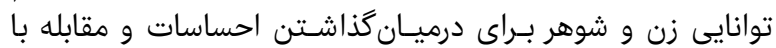

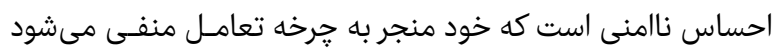

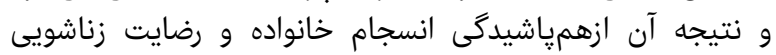

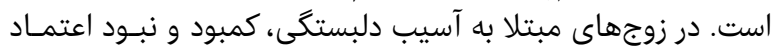

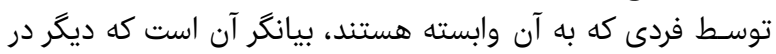

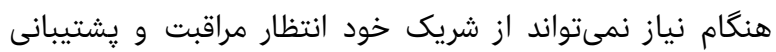

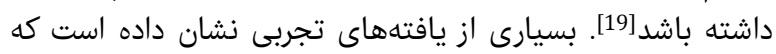

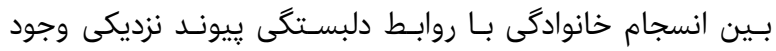

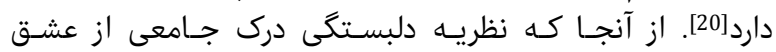

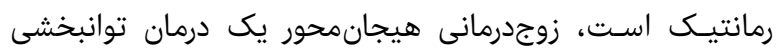

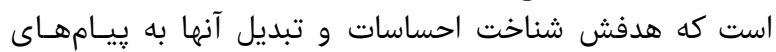

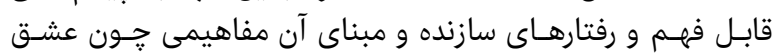

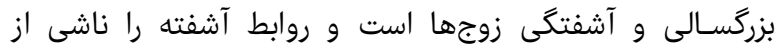

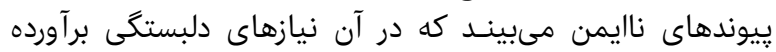

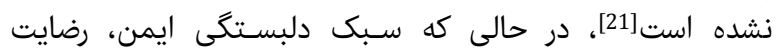

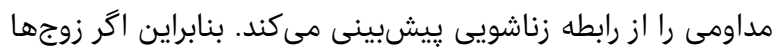

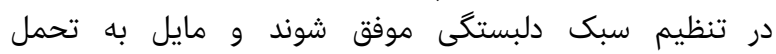

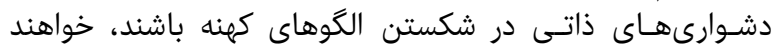

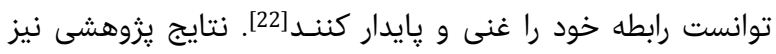

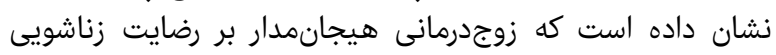

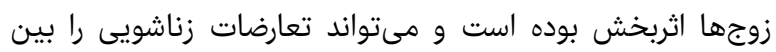

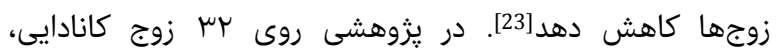

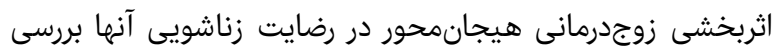

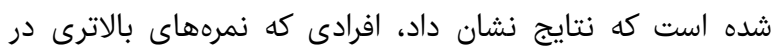

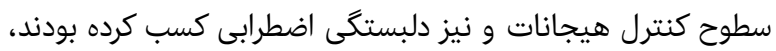

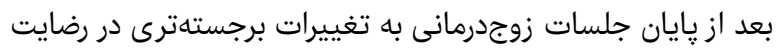

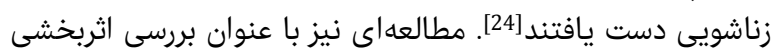

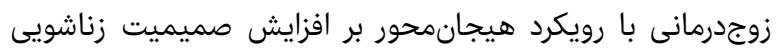

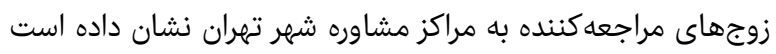

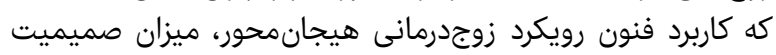

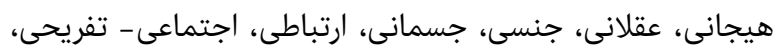

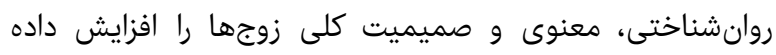

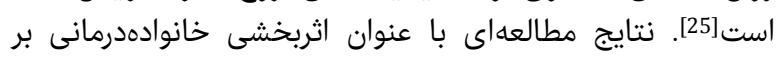

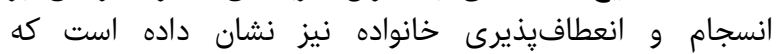

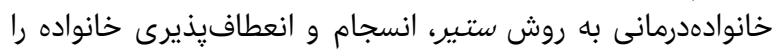

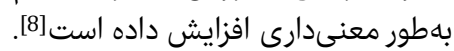

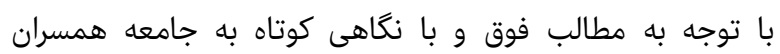

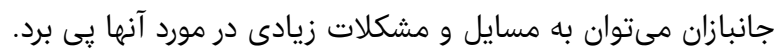

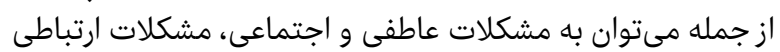

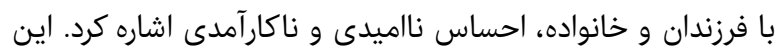

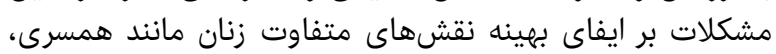

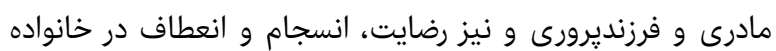

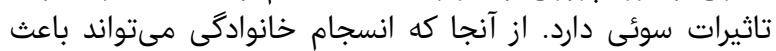

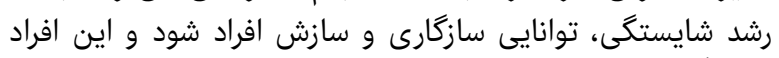

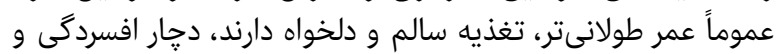

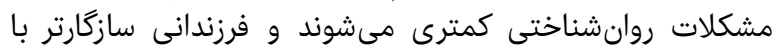

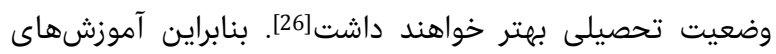

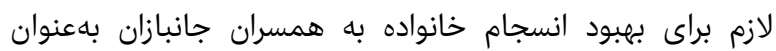


اجراى يُوهش و جلب رضايت آزمودنىها با تاكيد بر محرمانهبودن

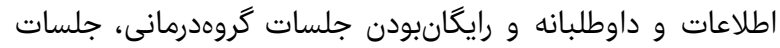

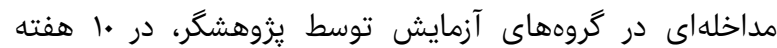

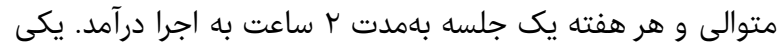

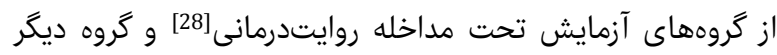
تحت مداخله درمان هيجانمحور [29] قرار گرفتند (جدول آنداني ().

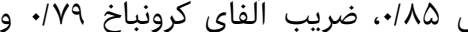

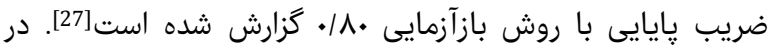

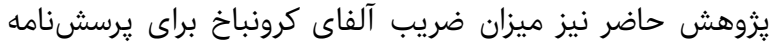

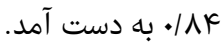

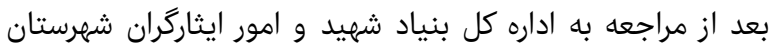

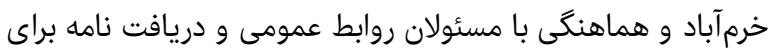

جدول 1) شرح جلسات درمانى روايتدرمانى و هيجانمدار براى همسران جانباز شهرستان خرمآباد (هر گروه •r نفر) شرح جلسات

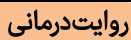

جلسه اول

توضيح رويكرد روايتدرمانى و راهكارهاى مربوط به آن، تشريح مفهوم انسجام خانواده

جلسه دوم تورئر

ترسيم و گَسترش داستان زندگى و دادن نقطه شروع به آن، آغاز برونسازى و مشخصسازى داستان و جكَونكى ساخت آنها در افراد

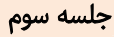

دستيابى به اصول حاكم بر داستانگ䧄 افراد، بررسى استعارههاى افراد و ديدن داستانهاى زندگى افراد از منظر ديكران

جلسه جهارم

دادن راهبردهاى لازم براى بى اثركردن مشكل و بررسى تاريخهِ بهوجودآمدن مشكل، استفاده از زبان برونسازى و جداسازى مشكل از هويت و ارتباطات مراجع، بررسى

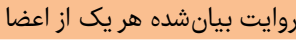

جلسه ينجم

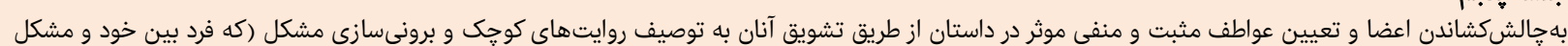

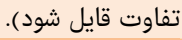

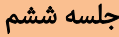

تشويق مراجع به روشنسازى عكسالعمل و عقايدش در مقابل مشكلات و اتفاقات از طريق يرسشهاى تاثيرگذار، بهإلشكشيدن داستان مشكلساز و برونىكردن مشكل جلسه هفتم

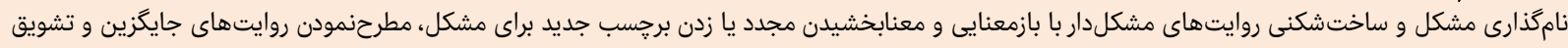

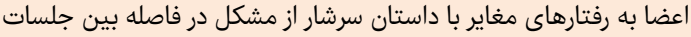

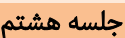

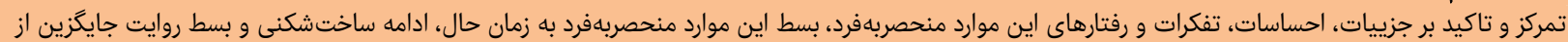

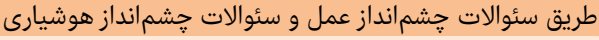
جلسه نهم ايجاد روايت جديد، درخواست از اعضا براى نوشتن نامههاى يستنشده، بخشيدن، فراموشكردن يا يا رهاكردن افراد زورگو و سوءاستفادهننده و كسانى كه در كذشته موجب خاطرات ناخوشايند براى فرد بودهاند.

جلسه دهم مرور خاطرات و اقدامات احتمالى اعضا درباره مطالب جلسه قبل، گفتتوظو در مورد تجربههاى مثبت و روايتهاى جايكزين، دوبارةكويى و تثبيت روايتهاى جايكزين، تعيين زمان يسآزمون

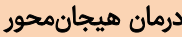

جملسه اول تعان

كشف تعاملات مشكلدار، ارزيابى شيوه برخورد آنها با مشكل، كشف موانع دلبستكى و دركيرى هيجانى درونفردى و بين فردى، سنجش وضعيت ارتباط زناشويى و انسجام

جلسه دوم تران

بررسى جرخه تعاملات زوجين، كشف إز إنه حوادث برجسته، كشف اطلاعاتى كه در حضور همسر امكانيذير نيست مانند ارزيابى ميزان تعهد نسبت به ازدواج، روابط فرا زناشويى، ، ارزيابى ميزان ترس آنها از افشاى رازها كوريا

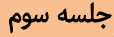
مشخصكردن الكوهاى تعامل شامل يذيرش احساس تصديقشده، كشف نايمنى دلبستكى و ترسهاى همسران، كمك به بازتربودن و خودافشايى همسران

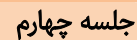
بازسازى بيوند زوجين شامل روشنكردن باسخهاى هيجانى كليدى، گسترش تجربه هيجانى هر كدام از مراجعان در رابطه و بررسى و تجديدنظركردن روابط

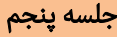
تخليه هيجان، عمقبخشيدن به دركيرى عاطفى زوجين بر مبناى دلبستخى، بهبود وضعيت درونروانى و بهبود وضعيت تعاملى

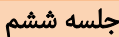
شناسايى ترسهاى زيربنايى، گستردمردن خود در ارتباط با ديكران، ارتقاى روشهاى جديد تعامل، تمركز روى خود نه ديكرى

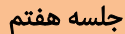
بازسازى تعاملات و تغيير رويدادها، دركيرى بيشتر زوجها با يكديكر، روشنسازى خواستهها و آرزوهاى مراجعان

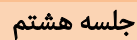
يافتن راه حلهاى جديد براى مشكلات قديمى شامل بازسازى تعاملات، ايجاد هماهنكى در حس درونى نسبت به خود و نيز رابطه و غيره

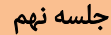
استفاده از دستاوردهاى درمانى در زندگى روزانه شامل شناسايى و حمايت از الخوهاى سازنده تعاملى، ايجاد دلبستخى ايمن، ساختن يك داستان شاد از رابطه جلسه دهم تعيين تفاوت بين الكَوى تعامل منفى گذشته در جلسات اوليه و اكنون، حفظ درگيرى هيجانى بهمنظور ادامه قوىكردن ييوند بين آنها 


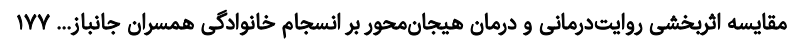

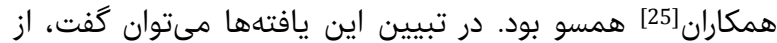

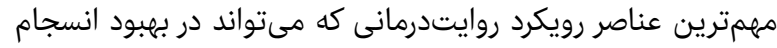

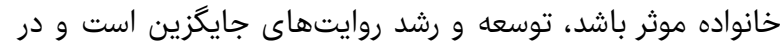

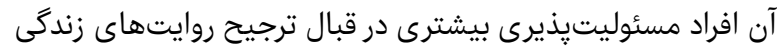

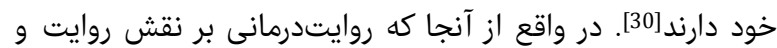

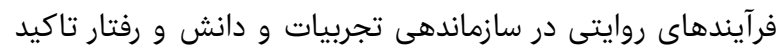

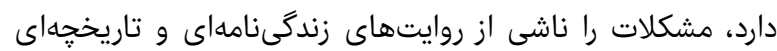

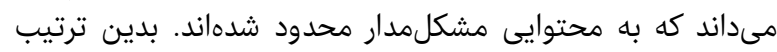

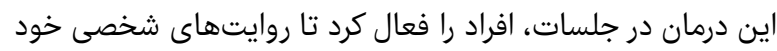

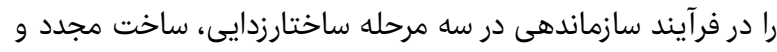

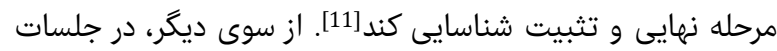

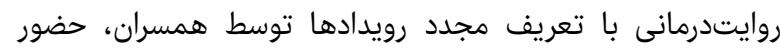

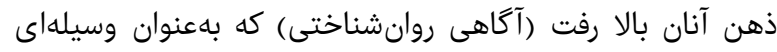

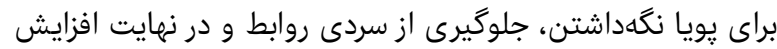

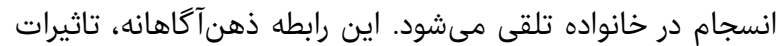

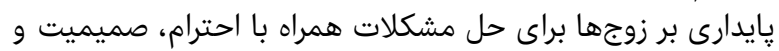

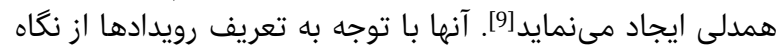

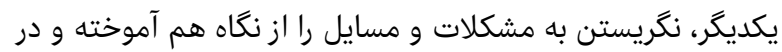

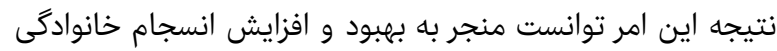
در آنان شود.

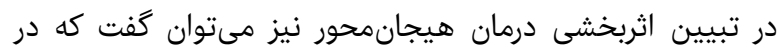

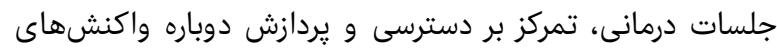

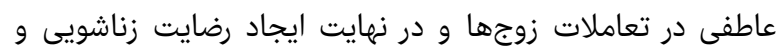

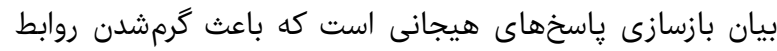

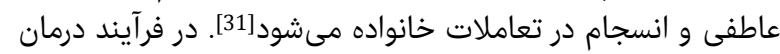

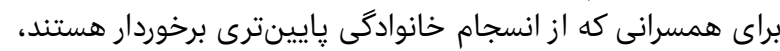

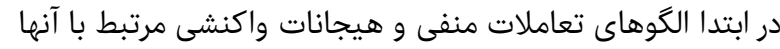

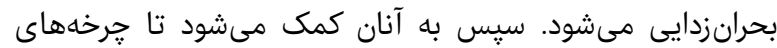

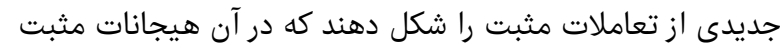

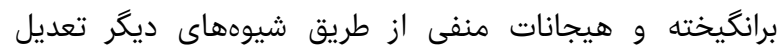

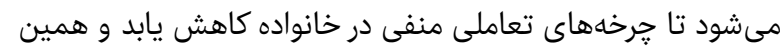

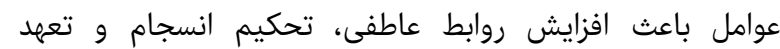

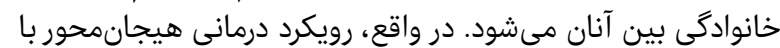

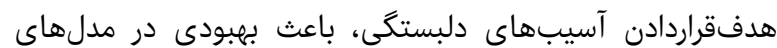

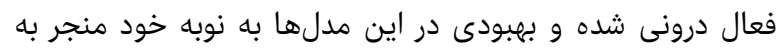

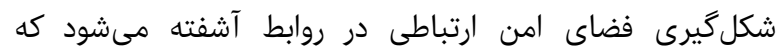

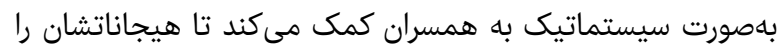

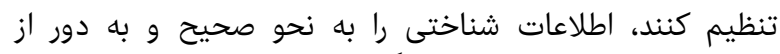

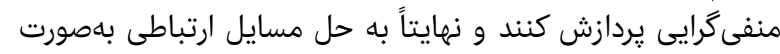

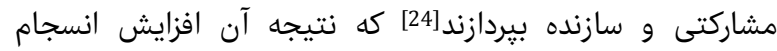

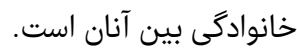

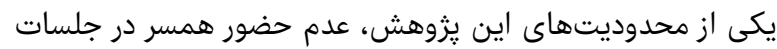

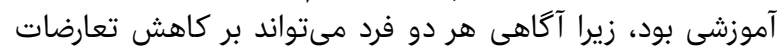

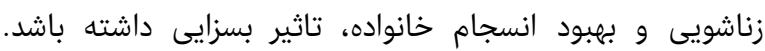

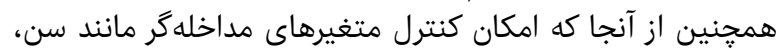

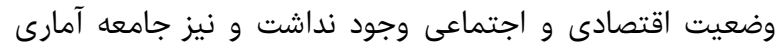

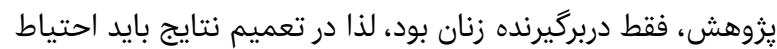

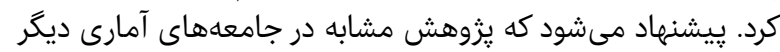

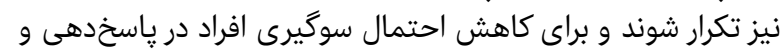

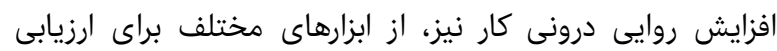

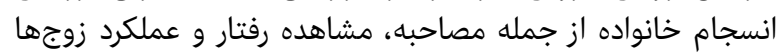

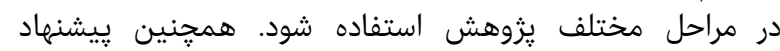

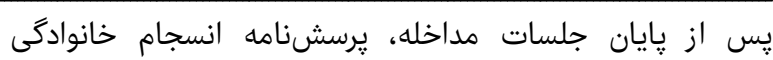

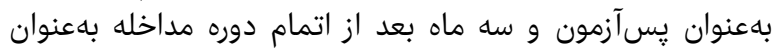

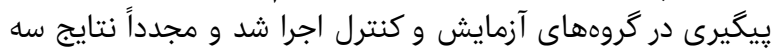

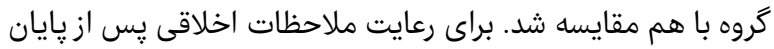

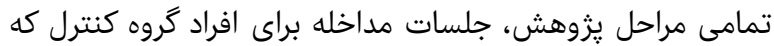

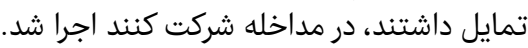

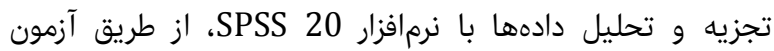

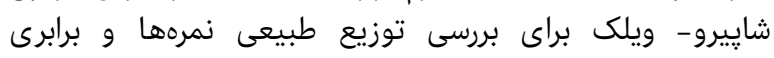

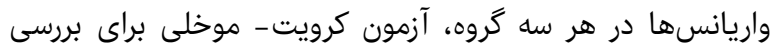

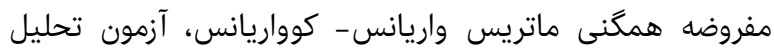

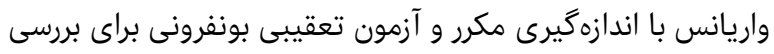

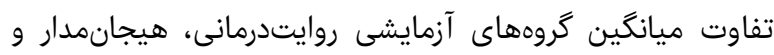
كروه كنترل صورت گرفت.

يافتهها ميانگين سنى همسران حانباز در گروه آزماش رواش روايتدرمانى ميانين سنى همسران جانباز در كروه آزمايش روايتدرمانى

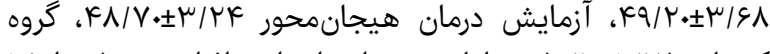

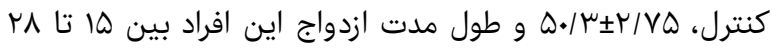

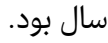
در يسآزمون و يِيگيرى، ميانگين نمرات دو گروه آزمايشى نسبت

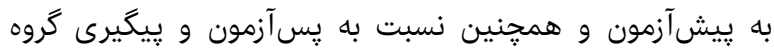

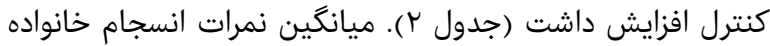

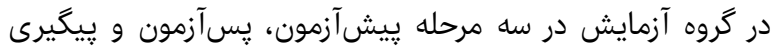

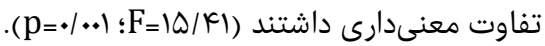

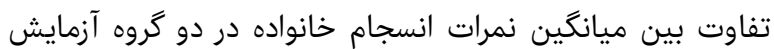

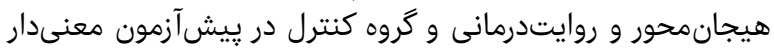

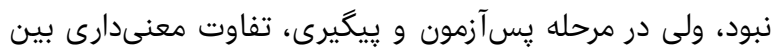

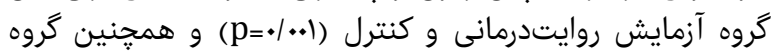

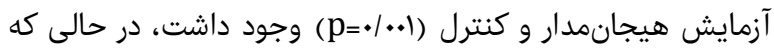

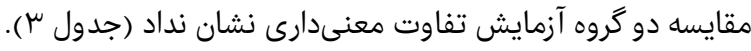

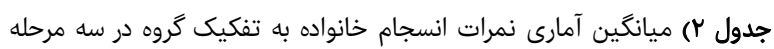

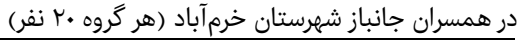

\begin{tabular}{|c|c|c|c|}
\hline ييگيرى & يسآزمون & پيش آزمون & گروه \\
\hline $\mathrm{\top} / / \Omega O \pm \mathrm{V} / \Lambda \mu$ & $V Q / \varepsilon \cdot \pm T / Y)$ & $T \varepsilon / r \cdot \pm r / q 1$ & \\
\hline$V / \mu \sigma \pm \mu / \mu V$ & $\wedge \mu / \vee \cdot \pm q / 7 \curlywedge$ & & درمان هيجانمح \\
\hline ז & $7 \mu / \% O \pm 0 / .9$ & $7 \mu / 1 \varepsilon \pm 0 / 10$ & كنترل \\
\hline
\end{tabular}

جدول سا) نتايج آزمون بونفرونى و اختلاف ميانكين حاصل از مقايسه جفتى إنى ميانكين نمرات انسجام خانواده همسران جانبان آنباز شهرستان خرمآباد آناد

\begin{tabular}{|c|c|c|c|}
\hline ييگيرى & يس آزمون & ييشآزمون & گروه \\
\hline$r / \Lambda Y \pm \varepsilon / r \Lambda$ & $-\mu / r \cdot \pm T / r O$ & $.10 \cdot \pm \mu / 1 r$ & روايتدرمانى - هيجانمحور \\
\hline$V / \varepsilon 0^{\circ 0} \pm \varepsilon / \mu \wedge$ & $M / I r^{\circ \circ} \pm V / R T$ & . $/ \varepsilon r \pm 0 / \mu$. & هيجانمحور-كنترل \\
\hline $0 / 79^{\circ 0} \pm \mu / \mu q$ & $10 / \varepsilon \Lambda^{\circ} \pm 7 / 7 \pi$ & $1 / \cdot r \pm \varepsilon / M \Lambda$ & روايتدرمانى_كنترل \\
\hline
\end{tabular}

بحث

يزوهش حاضر با هدف مقايسه اثربخشى روايتدرمانى و درمان

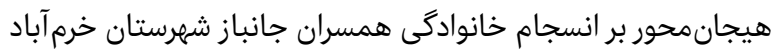

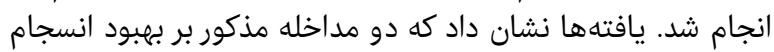

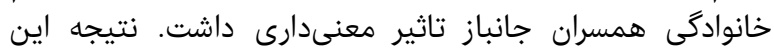

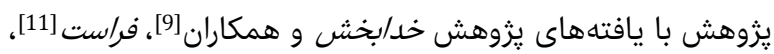

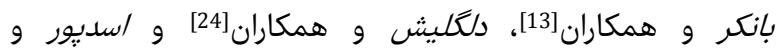


11- Frost DM. The narrative construction of intimacy and affect in relationship stories: Implications for relationship quality, stability, and mental health. J Soc Pers Relatsh. 2013;30(3):247-69.

12- Skerrett K. "Good Enough Stories": Helping couples invest in one another's growth. Fam Process. 2010;49(4):503-16.

13- Banker JE, Kaestle CE, Allen KR. Dating is hard work: A narrative approach to understanding sexual and romantic relationships in young adulthood. Contemp Fam Ther. 2010;32(2):173-91.

14- Changizi F, Panahali A. The effectiveness of group narrative therapy on the habitual life and happiness of the elderly in Tabriz. J Instr Eval. 2016;9(34):63-76. [Persian]

15- Rabiei S, Fatehizadeh M, Bahrami F. The effect of couple therapy narrative on family function of couples in Isfahan. Fam Res. 2008;4(14):179-91. [Persian]

16- Li T, Fung HH. How avoidant attachment influences subjective well-being: An investigation about the age and gender differences. Aging Ment Health. 2014;18(1):4-10. 17- Dillon DG, Ritchey M, Johnson BD, LaBar KS. Dissociable effects of conscious emotion regulation strategies on explicit and implicit memory. Emotion. 2007;7(2):354.

18- Bowlby J. A secure base: Parent-child attachment and healthy human development. New York: Basic books Press; 2008.

19- Johnson S, Zuccarini D. Integrating sex and attachment in emotionally focused couple therapy. J Marital Fam Ther. 2010;36(4):431-45.

20- Towler AJ, Stuhlmacher AF. Attachment styles, relationship satisfaction, and well-being in working women. J Soc Psychol. 2013;153(3):279-98.

21- Hollist CS, Miller RB. Perceptions of attachment style and marital quality in midlife marriage. Fam Relat. 2005;54(1):46-57.

22- Greenberg LS. Emotion-focused therapy. Clin Psychol Psychother. 2010;11(1):3-16.

23- Akhavan-Bitaghsir Z, Sanaee-Zaker B, Navabinejad Sh, Farzad V. The effectiveness of emotional focused couple therapy on satisfaction and marital adjustment in couples. Res Behav Sci. 2016;14(3):324-30. [Persian]

24- Dalgleish TL, Johnson SM, Burgess Moser M, Lafontaine MF, Wiebe SA, Tasca GA. Predicting change in marital satisfaction throughout emotionally focused couple therapy. J Marital Fam Ther. 2015;41(3):276-91.

25- Asadpour I, Nazari AM, Sanai Zaker B, Shaghaghi S. An investigation of effectiveness of the emotionally focused couple therapy on increasing marital intimacy in couples entered at counseling centers in Tehran. Res Clin Psychol Counsel. 2012;14(3):25-38. [Persian]

26- Cacioppo JT, Cacioppo S, Gonzaga GC, Ogburn EL, VanderWeele TJ. Marital satisfaction and break-ups differ across on-line and off-line meeting venues. Proc Natl Acad Sci. 2013;110(25):10135-40.

27- Samani S. Developing a family cohesion scale for Iranian adolescents. Presented in $28^{\text {th }}$ International Congress of Psychology. Beijing: Psychology Press; 2004. 28- White M, Epston D. Reflections on narrative practice: Essays and interviews. Adelaide: Dulwich Centre Press; 2000.

29- Johnson SM. The practice of emotionally focused marital therapy: Creating connection. 2nd Edition. New York: Brunner-Rutledge; 2004.

30- Bohlmeijer E, Kramer J, Smit F, Onrust S, van Marwijk $\mathrm{H}$. The effects of integrative reminiscence on depressive

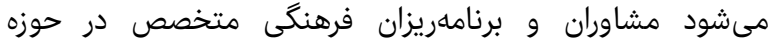

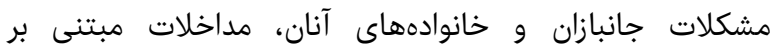

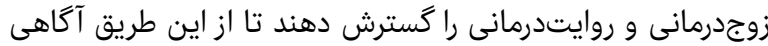

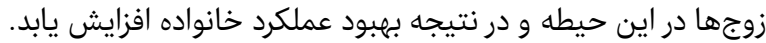

$$
\begin{aligned}
& \text { نتيجه گيرى } \\
& \text { روايتدرمانى و زوجدرمانى هيجانيرى محور بر بهبود انسجام خانواده } \\
& \text { همسران جانبازان موثر است. }
\end{aligned}
$$

تشكر و قدردانى: از سازمان بنياد شهيد و امور ايثارگران شهرستان

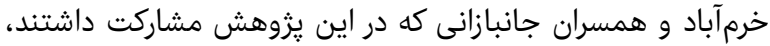
تشكر و قدردانى مى شود.

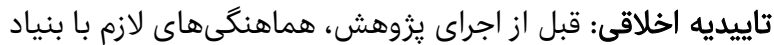

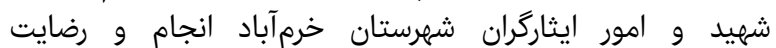

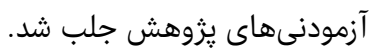

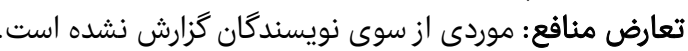

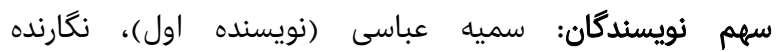

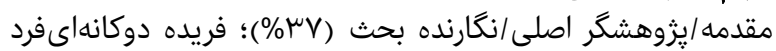

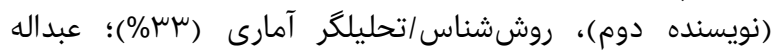

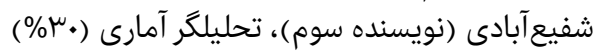

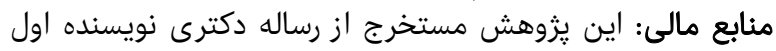

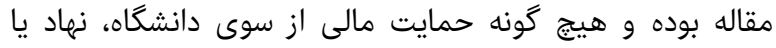
سازمانى انجام نكرفته است.

\section{منابع}

1- Yousefi A, Rafinia P, Sabahi P. The effectiveness of hope therapy on quality of life in wives of veterans with post traumatic stress disorder. J Clin Psychol. 2016;8(1):1-10. [Persian]

2- Gavlovski T, Lyons JA. Psychological sequellae of combat violence: A review of the impact of PTSD on the veteran's family and possible interventions. Aggress Violent Behav. 2004;9(5):477-501.

3- Figley CR. Stress disorders among vietnam veterans: Theory, research. 1 ${ }^{\text {st }}$ Edition. London: Routledge Press; 2014.

4- Soliemanian AA, Jajarmi M, Falahati M. Effectiveness of training life skills on the quality of life of spouses of veterans with affective disorders. Iran J War Public Health. 2015;7(4):197-205. [Persian]

5- Winek JL. Systemic family therapy: From theory therapy. $2^{\text {nd }}$ Edition. North Carolina: SAGE Press; 2010.

6- Mirnics Z, Vargha A, Tóth M, Bagdy E. Cross-cultural applicability of FACES IV. J Fam Psychother. 2010;21(1):17-33.

7- Olson D. FACES IV and the circumplex model: Validation study. J marital fam ther. 2011;37(1):64-80.

8- Bozorgmanesh K, Nazari AM, Zahrakar K. Effectiveness of family therapy on its cohesion and flexibility. J Holist Nurse Midwifery. 2016;26(4):27-35. [Persian]

9- Khodabakhsh MR, Kiani F, Noori Tirtashi E, Khastwo Hashjin $\mathrm{H}$. The effectiveness of narrative therapy on increasing couples intimacy and its dimensions: Implication for treatment. Fam Couns Psychother. 2015;4(4):607-32. [Persian]

10- Josselson R, Lieblich A, McAdams DP, editors. The meaning of others: Narrative studies of relationships. Washington: American Psychological Association; 2007. 
IV9 مقايسه اثربخشى روايتدرمانى و درمان هيجانمحور بر انسجام خانوادكى همسران جانباز... acceptance and commitment and emotionally focused couples therapy on improvement of intimacy and reduction of Alexithymia among Couples. Andishe va Raftar (Appl Psychol). 2017;11(44):47-56. [Persian] symptomatology and mastery of older adults. Community Ment Health J. 2009;45(6):476.

31- Zanganeh Motlag F, Bani-Jamali SS, Ahadi H, Hatami HR. The effectiveness of couples therapy based on 